\title{
Propuesta de un Tesauro de Patrimonio Histórico en Internet mediante Lenguaje ASP
}

\author{
$\mathrm{M}^{\mathrm{a}}$ José Escalona Cuaresma \\ David Márquez Salvago \\ Alfredo Martín Bonaño
}

Departamento de Lenguajes y Sistemas

Informáticos

Universidad de Sevilla

Antonio Martín Pradas

Área de Normalización Terminológica Centro de Documentación del IAPH

\begin{abstract}
Resumen
Este artículo presenta el resultado de un proyecto fin de carrera desarrollado en el Departamento de Lenguajes y Sistemas Informáticos de la Universidad de Sevilla, en colaboración con el Instituto Andaluz del Patrimonio Histórico (IAPH), en el que se ha elaborado un prototipo del Tesauro de Patrimonio Histórico en el lenguaje de programación ASP para su distribución en Internet.
\end{abstract}

Se analiza la necesidad de acercar el TPHA al público no experto en Patrimonio Cultural, presentándose una propuesta del mismo en Internet para lo cual ha sido desarrollada su interfaz, su estructura, así como su forma y manual de uso.

\section{Palabras Clave}

Tesauros / Patrimonio Histórico / Andalucía / Lenguajes documentales / Instituto Andaluz del Patrimonio Histórico / Internet / Lenguaje ASP

\section{SIPHA y el Tesauro de Patrimonio Histórico Andaluz (TPHA)}

Desde sus comienzos, el IAPH apostó por el uso de las nuevas tecnologías elaborando bases de datos que permitiesen gestionar la información patrimonial, naciendo así SIPHA. En un principio, estas bases de datos sólo podían ser consultadas en el Servicio de Información del Centro de Documentación del IAPH, así como en algunos organismos colaboradores. Sin embargo, y desde hace unos años, se ha producido un creciente interés por el patrimonio y la demanda de información desde muy distintos ámbitos se ha visto incrementada. Cada día son más los investigadores de patrimonio, las administraciones públicas y asociaciones en preservar el
Patrimonio Cultural, investigadores, usuarios en general y los turistas, todos ellos interesados en acceder a cualquier tipo de información relacionada con el Patrimonio. SIPHA debe dar soporte a todos estos usuarios por lo que actualmente está orientado a ser un sistema basado en una gran biblioteca digital, bastante compleja, que maneja una gran cantidad de información.

Con la creación del Centro de Documentación del $\mathrm{IAPH}$, siguiendo la política de conservación, investigación y divulgación del Patrimonio de Andalucía, se inició una primera etapa en la que se ponen en marcha una serie de Bases de Datos relacionadas con los bienes culturales autonómicos. En un principio las bases de datos eran independientes, observándose la necesidad de buscar la integración con miras a crear el Sistema de Información del Patrimonio Histórico Andaluz (SIPHA).

Esta integración se estableció por medio de dos instrumentos, por un lado la utilización de un mismo lenguaje documental y por otro la creación del SIPHA mediante una base de datos integrada.

Respecto a la utilización de un mismo lenguaje documental, el Centro de Documentación apostó por la realización del Tesauro de Patrimonio Histórico Andaluz (TPHA), proyecto que entrañaba un gran esfuerzo tanto en recursos humanos, así como económico y temporal.

El Centro de Documentación se inclinó por la realización de un Macrotesauro de Patrimonio Histórico, formando un Grupo de Trabajol integrado por representantes del IAPH, la Universidad y los Museos, siendo presentada la publicación en papel de la "Versión 0" en noviembre de 1998.

Este evento supuso un antes y un después en el tratamiento de la información y en la eficacia de su posterior recuperación. Hasta ese momento las bases de datos patrimoniales continuaban alimentándose, en mayor o en menor medida, del Avance del Tesauro. Tras la publicación del TPHA fue necesario realizar una serie de tablas de equivalencia entre los descriptores del Avance y el TPHA, normalizándose éstos en función del nuevo lenguaje documental.

Un problema que se detectó en el IAPH fue la multiplicidad de terminología utilizada para sistematizar la información. De esta forma, el usuario podría efectuar consultas en las bases de datos desde distintos puntos de búsqueda. Por esta razón, en el SIPHA se introdujo el TPHA. El Tesauro es utilizado por los catalogadores del IAPH a la hora de alimentar las bases de datos patrimoniales. Así, el Tesauro agrupa en una 
estructura jerárquica, de equivalencia y asociativa, los términos que se pueden introducir en determinados campos de las bases de datos. De esta forma, TPHA no es más que un conjunto de términos que se encuentran almacenados en una estructura, a modo de vocabulario controlado caracterizándose por su interdisciplinariedad. A medida que se va navegando y bajando de nivel en la estructura jerárquica, la información se hace más concreta, o sea se desciende de lo genérico hacia lo específico. El TPHA cuenta con I 4.387 descriptores normalizados, así como 1.376 entre sinónimos y cuasisinónimos, una gran cantidad de información estructurada en función de las diez facetas en las que se encuentra dividido el corpus del tesauro, aplicable a los distintos campos de las bases de datos; en muchos casos estas facetas se corresponden con los campos de las bases de datos a cumplimentar, como es el caso de Actividades, Estilo, Periodos geológicos. Periodos históricos, Materiales, etc., o en su caso encuentran una correspondencia como las Tipologías en Objetos inmuebles y Objetos muebles. De esta forma, cuando un catalogador da de alta un mueble o un inmueble lo puede hacer desde el punto de vista de cualquier disciplina relacionada directa o indirectamente con el Patrimonio Cultural. En las consultas, los catalogadores utilizan el Tesauro para encontrar aquellos términos que representan la información que desean encontrar, y lo utilizan para establecer los parámetros de sus consultas, con miras a que no se produzcan reverberaciones, ruidos innecesarios ${ }^{2}$ o silencios.

El Tesauro es un lenguaje vivo en constante evolución, por lo que tiene que ser revisado y ampliado, lo que le confiere varias características interesantes. Por un lado, contempla el uso de sinónimos y cuasisinónimos. De esta forma, si un mismo término tiene varios sinónimos, sólo uno de ellos puede ser utilizado, siendo éste el representante canónico.

La cantidad de términos del Tesauro y su complejidad estructural, así como el continuo cambio al que está sujeto, ha provocado que la opción del sistema local o el formato en papel del Tesauro no sea la solución más adecuada al problema. Además, al distribuir SIPHA mediante Internet, el Tesauro está a disposición de cualquier usuario del sistema.

\section{El Programa de gestión del TPHA}

La gestión del TPHA se realiza a través de una base de datos, Programa de Gestión de Tesauro, realizada expresamente para la construcción del mismo, permitiendo su utilización en el desarrollo de otros tesauros. En la actualidad cuenta con:

\footnotetext{
- 15.763 términos entre descriptores y no descriptores.

- 46 indicadores de faceta.

- 1.053 códigos alfanuméricos.

- 1.189 notas de alcance (NA).

- 13.220 relaciones entre descriptores (RT) ${ }^{3}$.
}

Es una aplicación concebida para la carga y gestión del Tesauro de Patrimonio Histórico Andaluz, realizada sobre un sistema de programación de entorno $\mathrm{Vi}$ sual Basic. Este software mantiene una estructura en módulos que permite efectuar con sencillez las funciones exigibles a un gestor de tesauros ${ }^{4}$.

Su estructura modular permite llevar a cabo distintas operaciones:

- Generación de la estructura de acuerdo a las normas internacionales, con operadores en castellano.

- Control de usuarios: usuarios invitados y usuarios autorizados.

- Consulta del Tesauro: siempre enfocada a usuarios.

- Modificación: con opciones de búsqueda de descriptores, colocación de notas de alcance, relaciones de equivalencia, relaciones asociativas, códigos alfanuméricos, códigos internos, indicadores de facetas, impresión de pantalla, etc.

- Administración: con opciones de chequeo, reorganización del código interno, gestión de usuarios y estadísticas. Las estadísticas muestran el número total de descriptores, no descriptores, indicadores de facetas, códigos alfanuméricos, notas de alcance, relaciones asociativas, etc.

- Listados: ver, generar e imprimir los listados que pueden ser de tipo jerárquico, alfasistemático, permutado y macroestructura de uno a tres niveles de profundidad, con la posibilidad de guardar los listados generados como ficheros de texto, gestionables desde cualquier procesador de texto.

Es un programa amigable y de gran utilidad para la navegación interna por el Tesauro, permitiendo una gestión ágil y eficaz ${ }^{5}$.

\section{Tesauro de Patrimonio Histórico en Internet (TPHI)}

Por todos los pros y los contras reseñados en el apartado anterior, se ha desarrollado una nueva aplicación para el TPH en Internet. Esta herramienta debe ofrecer la misma funcionalidad que el Tesauro publicado en papel de manera que:

- Debe presentar la estructura jerárquica, de equivalencia y asociativa, con el correcto tratamiento de las facetas, los términos genéricos y los términos específicos.

- Debe asegurar el tratamiento de sinónimos y notas de alcance.

- Debe contemplar varios tipos de búsquedas.

- Debe permitir el acceso de todo tipo de usuarios. De esta forma, distinguirá entre Administradores, que serán los que validen los nuevos descriptores y datos a incluir en el Tesauro y que básicamente se corresponde con el personal responsable del Tesauro en el IAPH; los Catalogadores, que serán lo que puedan proponer para añadir, borrar y modificar los diferentes descriptores y no descriptores del Tesauro; y los Usuarios Consultores, que podrán consultar el tesauro. 
A nivel informático, el Tesauro debe ofrecer una interfaz sencilla de manejar, amigable, para que cualquier persona, experto o no en informática y en Tesauros, pueda utilizarlo sin problemas. Además, el Tesauro debe estar desarrollado mediante un lenguaje de programación adecuado para su uso a través de Internet.

Todos estos aspectos se han cubierto en la aplicación desarrollada como propuesta para difundir el TPH en internet, tal y como se presenta a lo largo de este apartado.

\section{Interfaz del TPHI}

Está orientado a ofrecer una interfaz sencilla que permita realizar todas las operaciones pertinentes de una manera ágil y eficaz. El sistema ofrece tres interfaces diferentes, dependiendo del usuario que lo utilice en cada momento. Por ello, se va a presentar la interfaz dependiendo de quién se conecte. Para cualquier usuario, la primera pantalla que aparece es la de solicitud de clave. Los usuarios consultores no necesitan clave, así que basta con pulsar el botón "Consultar", el resto, los administradores y catalogadores deberán disponer de una clave para entrar que será dada por el IAPH.

A partir de aquí, el sistema analiza qué tipo de usuario se ha conectado y modifica la interfaz dependiendo del usuario.

La más sencilla es la realizada para el conjunto de Consultores. A éstos se les permite realizar dos tipos de consultas: búsquedas mediante texto y búsquedas mediante la estructura jerárquica.

La interfaz de búsqueda mediante texto es bastante amigable. El usuario introduce el texto que quiere buscar e indica los criterios de búsqueda. El sistema devolverá los términos que se parezcan al introducido por el usuario o que comiencen o terminen por las palabras parecidas, a modo de coincidentes.

Si el usuario selecciona la opción de incluir sinónimos, buscaría además en el grupo de sinónimos del tesauro. Esto se utiliza cuando el usuario no tiene la certeza de que está usando el representante canónico.

La búsqueda por la estructura jerárquica es más senciIla. Pulsando sobre la letra en cuestión le aparecen todos los términos que empiezan por la letra seleccionada, accediéndose a través de ella a consultar la estructura jerárquica.

La interfaz para los catalogadores va a permitir diversa funcionalidad:

- El alta de facetas, con genéricos y específicos.

- El alta de sinónimos para genéricos o específicos.

- Cualquiera de las consultas vistas para los consultores.
En el IAPH, los catalogadores proponen al Área de Normalización Terminológica los nuevos términos candidatos a formar parte del TPHA. Al ser un sistema que afecta a muchos usuarios, los que pueden aceptar estas altas, realizar cambios en la estructura de la jerarquía o realizar correcciones son los encargados de la gestión del tesauro. Por ello, la funcionalidad de los administradores contempla la funcionalidad de los catalogadores e incluye la posibilidad de:

- Añadir nuevos descriptores.

- Añadir nuevos sinónimos.

- Añadir notas de alcance.

- Aumentar la estructura horizontal mediante la colocación de Términos relacionados TR (Estructura asociativa).

- Eliminar descriptores, sinónimos, notas de alcance y TR.

- Cambiar microfacetas de la estructura jerárquica.

- Realizar correcciones ortográficas sobre descriptores, sinónimos, Notas de alcance, etc.

- Reasignar sinónimos o modificar la estructura jerárquica.

La interfaz del administrador va a seguir la misma filosofía que la anterior y visualmente es muy similar. Su diferencia radica en la funcionalidad que ofrece.

\section{Arquitectura del TPHI en Internet}

Tras analizar las diferentes posibilidades arquitectónicas que podían usarse para desarrollar TPHI, se ha optado por el lenguaje ASP (Active Server Pages) ${ }^{6}$. Esta arquitectura se caracteriza por estar orientada a Internet y ha dado muy buenos resultados en el trabajo con bases de datos en tiempo de respuesta tanto en la consulta como en la modificación.

La base de datos, desarrollada mediante Access 2000, contiene toda la información del tesauro. IIS (Internet Information Server) es la aplicación que Microsoft ofrece para la publicación de páginas web desarrolladas mediante ASP. De esta forma, cuando un usuario remoto realiza una operación, ésta llega a Internet e IIS se encarga de analizar la petición y actuar sobre la base de datos como sea necesario.

ASP tiene una ventaja esencial en este sistema y es que se ejecuta desde el lado del servidor. Cuando un usuario solicita, por ejemplo, una consulta llega a IIS que la ejecuta sobre la base de datos y prepara la respuesta. Esta respuesta llega al usuario en formato HTML, por lo que el sistema y el navegador del usuario no tiene problemas en entenderlo. Lo que permite además que el código de la aplicación de $\mathrm{TPHI}$ nunca llegue al usuario y se mantenga segura.

Sí hay que destacarle una desventaja sustancial a ASP, y es que es un producto Microsoft, lo que obliga a usar IIS y por tanto el sistema Windows en el servidor. Sin embargo, ofrece una rápida respuesta a los usuarios, puesto que se descarga el trabajo que los equipos de estos deben realizar, ya que lo que reciben es HTML y éste es fácil de visualizar y entender 
por cualquier navegador. Y por supuesto, no exige que los usuarios tengan nada instalado en sus ordenadores, excepto un acceso a Internet y un navegador.

\section{Ventajas e inconvenientes del TPHI en Internet}

El desarrollo de TPHI fue terminado en febrero de 2002 y actualmente está siendo analizado en el IAPH. Aunque ya está publicado en Internet7 todavía no ha sido totalmente utilizado. Sin embargo, ya se le pueden destacar algunas ventajas:

I. Los usuarios pueden consultar y trabajar con TPHI desde cualquier parte del mundo, cosa que antes con el Tesauro en local o en papel era imposible.

2. TPHI ofrece todas las posibilidades que ofrecía el Tesauro en papel, haciendo facilitando su gestión en líneas generales.

3. TPHI tiene todas las ventajas que tiene cualquier Tesauro, permitiendo que cualquier usuario utilice los mismos términos para designar un mismo concepto.

4. TPHI, al gestionarse por Internet, mantiene en todo momento la información actualizada y al día, aspecto que era imposible mantener en la versión en papel o en local.
Pero a TPHI se le puede resaltar una importante desventaja y es el hecho de que los usuarios consultores no deberían usar el tesauro de forma directa. Esto es, tal y como el tesauro se encuentra actualmente es muy útil para catalogadores y administradores, que conocen su existencia y estructura. Ahora bien, a pesar de que la interfaz planteada es sencilla, ofrecer el Tesauro de Patrimonio Histórico así a los consultores es sólo un paso intermedio.

Actualmente, el Tesauro es autónomo y funciona de forma independiente al resto del SIPHA. Lo ideal sería que cuando un turista pidiese información al SIPHA, éste usara la funcionalidad del tesauro de manera que si el turista dice estar interesado en Pintura barroca, pues el sistema no sólo busca este término, sino que usa el tesauro y devuelve sus genéricos y específicos de la misma forma que se puede buscar en el tesauro.

Hoy en día, el problema ha sido parcialmente resuelto introduciendo cajas de listas con descriptores del Tesauro en los campos requeridos. Pero en un futuro lo ideal sería integrar el Tesauro completamente en el SIPHA haciéndolo transparente al usuario no experto. Aunque eso sí, manteniendo la posibilidad de trabajar con él a usuarios, catalogadores y administradores.

\section{Referencias}

BAENA PADILLA, E; CANCA CUENCA, 」, y MARTÍN PRADAS, A.: "El Programa de Gestión del tesauro de Patrimonio Histórico Andaluz". En PH Boletín del IAPH. Año VI, n² 24, septiembre 1998, p. II0-116.

BRISABOA, N. R.; OCAÑA, E.; PENABAD, M. R.; PALACES, A. S. y RODRÍGUEZ, F.j:: "Biblioteca Virtual de Literatura Gallega". Ideas 2002. Cuba, abril 2002.

CORDERO, J. M.; ESCALONA, Ma J.; TORRES, J.; MEJÍAS, M. y GASCA, R. M.: "Aplicación de los sistemas de tratamiento de bibliotecas digitales a la gestión de Patrimonio Histórico". Número monográfico. Estudios Turísticos No 146. Madrid: Instituto de Estudios Turísticos, diciembre 2000, p. 37-47.

GASCA, R. M.; ESCALONA, Ma J.; ORTEGA, J. A.; MEJÍAS, M. y TORRES, J.: "Aplicación de la programación con restricciones a la elaboración automática de itinerarios culturales". Congreso de Turismo y Tecnologías de la Información y las Comunicaciones: Nuevas Tecnologías y Patrimonio. Madrid, octubre 2001.

GONZÁLEZ, M.; MEJÍAS, M.; ESCALONA, Ma J.; MARTÍNEZ y ORTEGA, J. A.: "Interacción con los Usuarios en bibliotecas digitales". I Jornadas Dolmen. Sevilla, junio 2001.

HILLIER, S. y MEZICK, D.: Programación de Active Server Pages. Mcgraw Hill, 2001.
INSTITUTO Andaluz de Patrimonio Histórico. Dirección General de Bienes Culturales. Consejería de Cultura. Junta de Andalucía. www.iaph.junta-andalucía.es.

MAJÓ, M.: "Los Sistemas de Información en los Destinos Turísticos". Turitec 2001. Madrid, octubre 2001.

MÁRQUEZ SALVAGO, D. y MARTÍN BONAÑO, A.: Tesauro de Patrimonio Histórico en Internet http://www25.brinkster.com/tesauro.

MARTíN PRADAS, Antonio: "Hablando un mismo idioma. El Tesauro del Patrimonio Histórico y su aplicación en los distintos ámbitos patrimoniales". En PH Boletín del IAPH. Año VIII, n 31 , marzo 2000, p. 106-1 II.

MARTÍN PRADAS, Antonio: "El lenguaje documental del Sistema de Información del patrimonio Histórico Andaluz: El Tesauro de Patrimonio Histórico Andaluz". En I Jornadas de Documentación. Sistemas y políticas de Información en el Estado de las Autonomías. Sevilla: AAD, 1997, p. 106-1।1.

TESAURO de Patrimonio Histórico Andaluz. Versión 0. Granada: Junta de Andalucía, 1998, p. 33-34.
I. MARTÍN PRADAS, Antonio: "Hablando un mismo idioma. El Tesauro del Patrimonio Histórico y su aplicación en los distintos ámbitos patrimoniales". En PH Boletín del IAPH. Año VIII, n 31 , marzo 2000, p. 106-III.

2. Ibídem.

3. INSTITUTO Andaluz del Patrimonio Histórico. Dirección General de Bienes Culturales. Consejería de Cultura. Junta de Andalucía. www.iaph.junta-andalucia.es

4. TESAURO de Patrimonio Histórico Andaluz. Versión 0. Granada: Junta de Andalucía, 1998, p. 33-34.
5. MARTÍN PRADAS, Antonio: "El lenguaje documental del Sistema de Información del patrimonio Histórico Andaluz: El Tesauro de Patrimonio Histórico Andaluz". En I Jornadas de Documentación. Sistemas y políticas de Información en el Estado de las Autonomías. Sevilla: AAD, p. I06-I I I.

6. HILLIER, S. y MEZINCK, D.: Programación de Active Server Pages. Mcgraw Hill. 2001

7. MARTÍN BONAÑO, A. y MÁRQUEZ SALVAGO, D.: Tesauro de Patrimonio Histórico en Internet. http://www25.birnkster.com/tesauro. 